Using these estimates in (17), we see that

$$
\log |F(z)|=\log |f(z)|+\log \left|p_{n}(z)\right|-\log \left|1-\left(\frac{z}{r_{n}}\right)^{m_{n}}\right|+O(n) .
$$

Combined with the estimates (15) and (13) this shows that

$$
\log |F(z)| \sim N(r)
$$

outside small pits around the zeros.

\title{
REFERENCE
}

1. J. E. Littlewood and A. C. Offord, On the distribution of zeros and a-values of a random integral function. II, Ann. of Math. (2) 49 (1948), 885-952.

G. M. College, Sambalpur, India.

\section{APPROXIMATE FUNCTIONAL APPROXIMATIONS AND THE RIEMANN HYPOTHESIS}

\section{ROBERT SPIRA}

1. Introduction. Using the functional equation for the Riemann zeta function

$$
\zeta(s)=\chi(s) \zeta(1-s)
$$

where

$$
1 / \chi(s)=(2 \pi)^{-s} 2 \cos (\pi s / 2) \Gamma(s),
$$

it was shown in Spira [1] that

(3) $\zeta(s) \neq 0,1 / 2<\sigma<1, \quad t \geqq 10$ implies $|\zeta(1-s)|>|\zeta(s)|$

where $s=\sigma+i$. Using similar but improved techniques, Schoenfeld and Dixon [2] strengthened the result (3) to assuming only $\sigma>1 / 2$, $|t| \geqq 6.8$ and $\zeta(s) \neq 0$. It easily follows from this inequality that the Riemann hypothesis is equivalent to the inequality $|\zeta(1-s)|$ $>|\zeta(s)|, 1 / 2<\sigma<1, t \geqq 10$.

Consider now the formula for $\zeta(s)$ which gives rise to the approximate functional equation and the Riemann-Siegel formula:

Presented to the Society, January 24, 1966 under the title Zeros of approximate functional approximations; received by the editors August 6, 1965 and, in revised form, October 26, 1965. 


$$
\zeta(s)=g_{m}(s)+\frac{e^{i \pi s} \Gamma(1-s)}{2 \pi i} \int_{C} \frac{w^{s-1} e^{-m w}}{e^{w}-1} d w
$$

where

$$
g_{m}(s)=\sum_{n=1}^{m} n^{-s}+\chi(s) \cdot \sum_{n=1}^{m} n^{s-1} .
$$

The $g_{m}(s)$ are the approximate functional approximations of the title, and, as noted implicitly by Titchmarsh ([4, p. 74]), they satisfy the same functional equation as $\zeta(s)$. Hence, just as in the case of the $\zeta$-function, $g_{m}(s)$ has its zeros on the critical line for $|t|>6.8$ if and only if $\left|g_{m}(1-s)\right|>\left|g_{m}(s)\right|$.

It is thus natural to write (4) in the form

$$
\zeta(s)=g_{m}(s)+B
$$

and study the location of the zeros of $g_{m}(s)$, (hopefully on the critical line), and attempt to carry the final conclusion of the Riemann hypothesis via the ideas of Rouché.

It is indeed possible to show that $g_{1}(s)$ and $g_{2}(s)$ have their zeros on the critical line (for $t$ sufficiently large) and this proof is carried out in $\$ 3$, with the aid of two lemmas in $\$ 2$.

Massive calculations were undertaken to verify the hypothesis for $m \geqq 3$, but these calculations instead revealed a remarkable scientific situation, which reinforces the possibility of using Rouché's theorem. The evidence strongly suggests the conjecture: If $m \geqq 3$, and $s$ is in the critical strip, then $g_{m}(s)$ has its zeros on the critical line for $(2 \pi m)^{1 / 2} \leqq t \leqq 2 \pi m$, and has zeros off the line outside this interval. The computations supporting this conjecture will be reported in full in another paper.

2. Lemmas on $\chi(s)$. We write $D$ for $d / d s$ and $D_{\sigma}$ for $\partial / \partial \sigma$.

Lemma 1. If $|t| \geqq 10$ and $\sigma>1 / 2$ then $D_{\sigma} \log |1 / \chi(s)|>\log |s|-1.93$.

Proof. From Schoenfeld-Dixon [2], we have $D_{\sigma} \log |1 / \chi(s)|$ $>\log |s|-|s|^{-1 / 2}-|s|^{-2} / 12-|t|^{-3} / 5-\left(\log 2 \pi+\pi /\left(4 \sinh ^{2}(\pi t / 2)\right)\right)$ from which the lemma easily follows.

LEMMA 2. If $|t| \geqq 10$ and $\sigma>1 / 2$ then

$$
|1 / \chi(s)|>.9646(|s| /(2 \pi))^{\sigma-1 / 2} \text {. }
$$

Proof. We have

$$
|1 / \chi(s)|=|2 \pi|^{-\sigma}|2 \cos (\pi s / 2)||\Gamma(s)| \text {. }
$$


As shown in Spira [1],

$$
|2 \cos (\pi s / 2)| \geqq 2 \sinh (\pi t / 2)=e^{\pi t / 2}-e^{-\pi t / 2}>.99 e^{\pi t / 2},
$$

the last inequality holding for $t \geqq 10$. Also from Spira [1] we have

$$
|\Gamma(s)|=(2 \pi)^{1 / 2} e^{-\sigma}|s|^{\sigma-1 / 2} e^{-t \text { arg } s}\left|e^{1 /(12 s)+R_{1}}\right|
$$

where $\left|R_{1}\right|<|s|^{-1} / 6$. It is easy to see that if $|z|<1$, then $\left|e^{z}\right| \geqq 1$ $-|z|[1 /(1-|z|)]$. Now $\left|1 /(12 s)+R_{1}\right|<|s|^{-1 / 12}+|s|^{-1} / 6=|s|^{-1 / 4}$ $\leqq 1 / 40$ if $t \geqq 10$. Hence, setting $z=1 /(12 s)+R_{1}$,

$$
\left|e^{1 /(12 s)+R_{1}}\right| \geqq 1-|z|[1 /(1-|z|)] \geqq 38 / 39
$$

the last inequality holding since $|z|<1 / 40$. By elementary geometry $t(\pi / 2-\arg s)>\sigma$, so the lemma followson combining equations (6)-(9).

\section{The cases $m=1,2$.}

TheOREM For $m=1,2$ and $|t|$ sufficiently large, $g_{m}(s)$ has all its complex zeros on $\sigma=1 / 2$.

Proof. For $m=1$ we have $g_{m}(s)=1+\chi(s)$, and for $\sigma>1 / 2$ and $|t|>6.8$, by Schoenfeld-Dixon [2], we have $\left|g_{1}(s)\right| \geqq 1-|\chi(s)|>0$. An easy argument shows that $g_{1}(s)$ has exactly one zero in each Gram interval.

For $m=2,\left|g_{m}(s)\right| \geqq\left|1+2^{-s}\right|-|\chi(s)| \cdot\left|1+2^{s-1}\right|$, and $\left|g_{2}(s)\right|>0$ provided

$$
|1 / \chi(s)|>\left|\left(1+2^{s-1}\right) /\left(1+2^{-s}\right)\right| \text {. }
$$

On $\sigma=1 / 2$ both sides of (10) are 1 , so that proceeding as in Schoenfeld-Dixon [2], (10) will hold provided

$$
D_{\sigma} \log |1 / \chi(s)|>D_{\sigma} \log \left|\left(1+2^{s-1}\right) /\left(1+2^{-s}\right)\right| \text {. }
$$

Since (Schoenfeld-Dixon [2]) $D_{\sigma} \log |f(s)|=\operatorname{Re} D \log f(s)$,

$$
\begin{aligned}
D_{\sigma} \log \left|\frac{1+2^{s-1}}{1+2^{-s}}\right| & =\log 2 \operatorname{Re}\left[\frac{1+2^{-s}+2^{s-1}}{\left(1+2^{-s}\right)\left(1+2^{s-1}\right)}\right] \\
& \leqq \log 2\left\lfloor\frac{1+2^{-s}+2^{s-1}}{\left(1+2^{-s}\right)\left(1+2^{s-1}\right)}\right\rceil \\
& \leqq \log 2\left[\frac{1+2^{-\sigma}+2^{\sigma-1}}{\left(1-2^{-\sigma}\right)\left(1-2^{\sigma-1}\right)}\right]
\end{aligned}
$$

where we must now take $1 / 2<\sigma<3 / 4$ to obtain a bound on the denominator. The numerator $1+2^{-\sigma}+2^{\sigma-1}$ has a minimum at $\sigma=1 / 2$, 
and for $1 / 2<\sigma<1$ rises monotonely from $1+(2)^{1 / 2}$ to 2.5 . The denominator is $1.5-\left(2^{-\sigma}+2^{\sigma-1}\right)$ which is smallest at $\sigma=3 / 4$. Thus $D_{\sigma}\left|\log \left(1+2^{s-1}\right) /\left(1+2^{-8}\right)\right|<2.5 \log 2 /\left[1.5-\left(2^{-3 / 4}+2^{-1 / 4}\right)\right]<27$. Using Lemma 1 , we need only choose $|s|$ so large that $\log |s|>1.93$ +27 , i.e., $t>e^{29}$.

For $\sigma>3 / 4$, we proceed directly from (10) using Lemma 2 . We have $\left|\left(1+2^{s-1}\right) /\left(1+2^{-s}\right)\right| \leqq\left(1+2^{\sigma-1}\right) /\left(1-2^{-3 / 4}\right)$ so that $(10)$ will hold provided

$$
.9646(|s| /(2 \pi))^{\sigma-1 / 2}>\left(1+2^{\sigma-1}\right) /\left(1-2^{-3 / 4}\right) .
$$

For $3 / 4 \leqq \sigma \leqq 1$, the right hand side of $(12)$ is bounded by 5 , and an easy calculation shows we need only take $t>2 \pi \cdot 6^{4} \sim 8145$. For $\sigma>1$, $1+2^{\sigma-1}<2^{\sigma}$, so (12) transforms to $(|s| / 4 \pi)^{\sigma-1 / 2}>(2)^{1 / 2} / .376$, which will be valid if $t>4 \pi\left((2)^{1 / 2} / .376\right)^{4} \sim 805$. This completes the proof of the theorem of this section.

Since there is empirically a steady appearance of zeros off the critical line for $m \geqq 3$, it appears unlikely that one would be able to extend the theorem of this section to any further $m$.

\section{REFERENCES}

1. Robert Spira, An inequality for the Riemann zeta function, Duke Math. J. 32 (1965), 247-250.

2. R. D. Dixon and Lowell Schoenfeld, On the size of the Riemann zeta-function at places symmetric with respect to the point $1 / 2$,Duke Math. J. (to appear).

3. D. H. Lehmer, Extended computation of the Riemann zeta-function, Mathematika 3 (1956), 102-108.

4. E. C. Titchmarsh, The theory of the Riemann zeta-function, Oxford at the Clarendon Press, 1951.

5. Robert Spira, Zeros of sections of the zeta function, (to appear).

University of TenNesseE 